\title{
Percutaneous Tumor Radioablation: A Case Report
}

\author{
Nagaraj Morubagal ${ }^{1}$, Sahajananda Hiremathada ${ }^{2}$, Manjula Nagaraj $^{3}$, Hirachand Muttagi ${ }^{4}$
}

\begin{abstract}
Osteoid osteoma is a benign bony tumor affecting children and young adults. Surgical excision, despite being the gold standard, is associated with morbidities and local recurrence, and radiofrequency ablation (RFA), being minimally invasive, is a viable therapeutic alternative.

Keywords: Minimally invasive technique, Osteoid osteoma, Radiofrequency ablation.

The Journal of Medical Sciences (2019): 10.5005/jp-journals-10045-00115
\end{abstract}

\section{Case Description}

A 23-year-old software professional presented with complaints of an axial thoracic back pain for a duration of 3 years. It was a dull aching type of pain, with visual analog scale (VAS) 7-9/10. The pain would worsen at night and did not show any variation with daily activities. There were no focal neurological deficits. The patient was referred to our clinic by neurosurgery to locate the source of the pain. Magnetic resonance imaging (MRI) of the thoracic spine showed an osteoid osteoma at the left D8 lamina.

The patient was posted for a left facet medial branch block at the D7-D8 level. Post-procedure, VAS scores (Fig. 1) improved from $10 / 10$ to $2 / 10$, an effect which lasted for 72 hours. After having been counseled about the risks and benefits of RFA vs open laminectomy and pedicle screw fixation, the patient consented to undergo the percutaneous RFA by impedance as the end-point method.

Under monitored anesthetic care, with full aseptic precautions, the procedure was performed with the patient in the prone position, using the Boston scientific equipment. D8 vertebra was squared and a $14 \mathrm{G}$ co-axis cannula was inserted between the $\mathrm{D} 8$ pedicle and the transverse process. An $18 \mathrm{G}$ RF cannula, with a $10-\mathrm{mm}$ active tip, was inserted through the co-axis cannula and the final position was confirmed by axial and coronal computerized tomography (CT) cuts (Fig. 2). After 2\% lignocaine infiltration, radiofrequency (RF) lesioning was done over 2 cycles ( 3.5 minutes with $5 \mathrm{~W}$ and 4.5 minutes with $5 \mathrm{~W}$ ). About $1 \mathrm{~mL}$ of $0.25 \%$ bupivacaine was deposited and pressure dressing was applied.

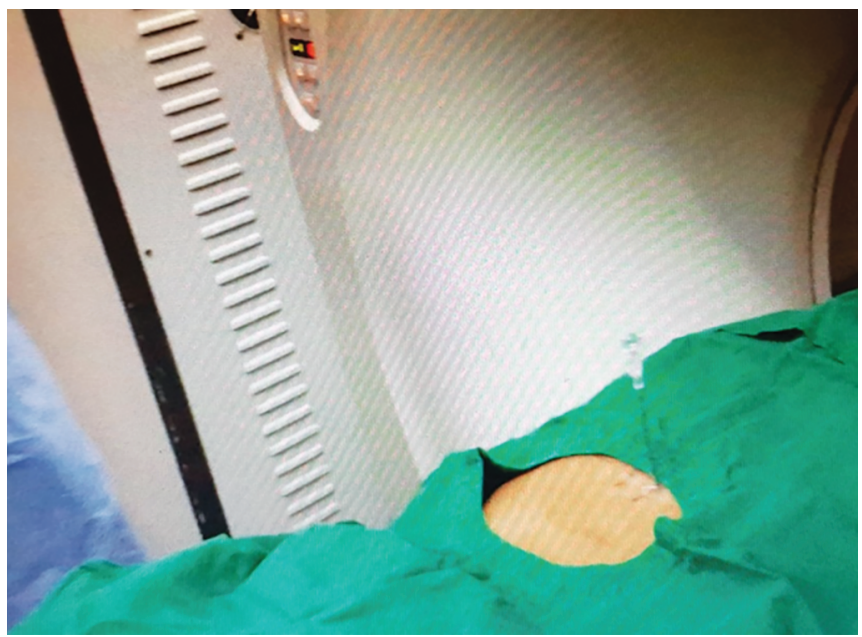

Fig. 1: Patient position
${ }^{1}$ Department of Anaesthesia and Pain Medicine, University Hospitals of Derby and Burton, UK

${ }^{2}$ Department of Anaesthesiology, RajaRajeswari Medical College and Hospital, Bengaluru, Karnataka, India

${ }^{3}$ Department of Surgery, RajaRajeswari Medical College, Bengaluru, Karnataka, India

${ }^{4}$ Department of Pain and Palliative Care, RajaRajeswari Medical College, Bengaluru, Karnataka, India

Corresponding Author: Sahajananda Hiremathada, Department of Anaesthesiology, RajaRajeswari Medical College and Hospital, Bengaluru, Karnataka, India, Phone: +91 9448085401, e-mail: sahaj_ anand@hotmail.com

How to cite this article: Morubagal N, Hiremathada S, et al. Percutaneous Tumor Radioablation: A Case Report. J Med Sci 2019;5(2):49-50.

Source of support: Nil

Conflict of interest: None

\section{RESULTS}

The patient experienced immediate postprocedural pain relief (VAS 0/10). Follow-up evaluation results at immediate postoperative period, 6 months, and 2 years are as follows: patients experienced less pain post-procedure (VAS of 1-2) and could sleep well after the procedure and did not require any medication for 48 hours (Fig. 3). During the subsequent follow-up visits (Fig. 4), they hardly

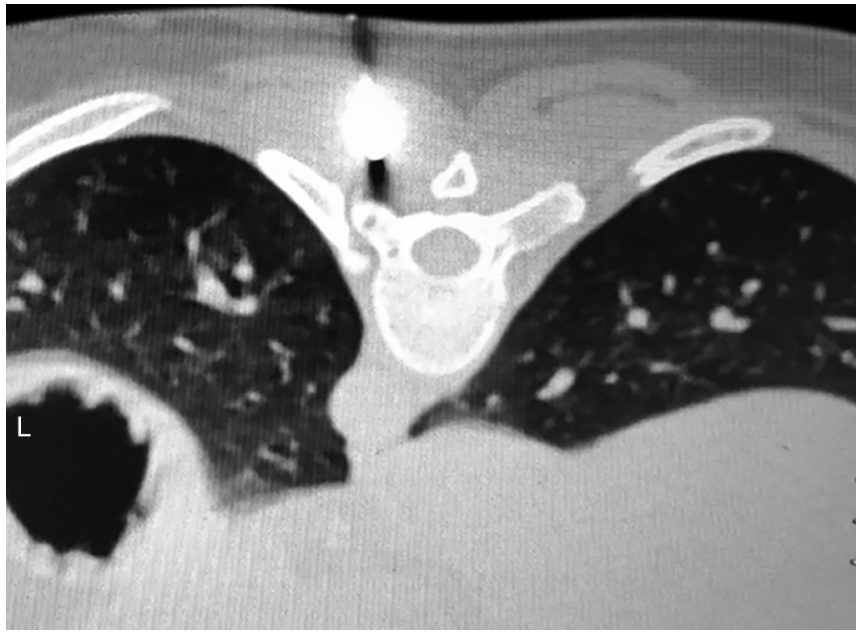

Fig. 2: CT showing RFA procedure at D8 vertebra

(O) The Author(s).2019 Open Access This article is distributed under the terms of the Creative Commons Attribution 4.0 International License (https://creativecommons. org/licenses/by-nc/4.0/), which permits unrestricted use, distribution, and non-commercial reproduction in any medium, provided you give appropriate credit to the original author(s) and the source, provide a link to the Creative Commons license, and indicate if changes were made. The Creative Commons Public Domain Dedication waiver (http://creativecommons.org/publicdomain/zero/1.0/) applies to the data made available in this article, unless otherwise stated. 


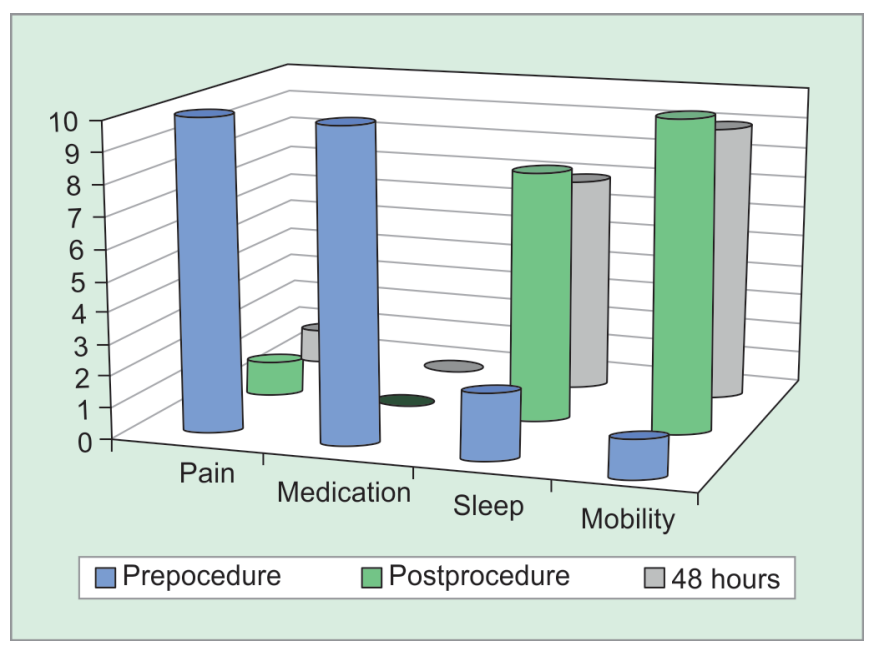

Fig. 3: Result of thoracic facet medial branch block

received pain medications and the same results were visible after 6 months and up to 2 years.

\section{Discussion}

Osteoid osteoma is a benign osteogenic tumor commonly affecting children and young adults. It accounts for almost $13.5 \%$ of all benign tumors. They commonly involve the diaphysis of the femur or the tibia but can occur anywhere in the skeleton. They present as nocturnal bony pain typically responsive to nonsteroidal anti-inflammatory drugs.

Surgical resection of the tumor has been the dictum for its management. But with the rise of the minimally invasive RFA technique, the disadvantages of the surgical approach have been circumvented, with similar success rates. We did not observe any major or minor complications during or after the procedure. Similar observations were made in a study by Upadhyaya et al. ${ }^{1}$

Kumar et al. conducted CT-guided RFA in 30 pediatric patients. They concluded that RFA is a safe and highly effective modality of treatment in the pediatric population, even in technically difficult locations. $^{2}$

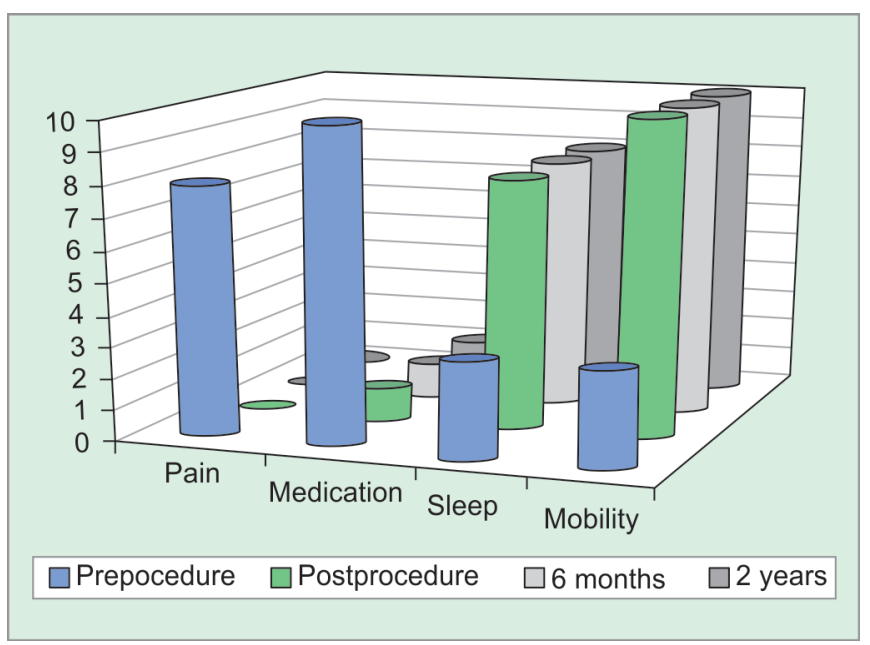

Fig. 4: 2 years follow-up evaluation of tumor radiofrequency ablation

Our patient has benefitted immensely from this minimally invasive intervention following a failed conservative management.

Due to its minimal invasiveness and comparable success rates to surgical excision, RFA is an attractive alternative ${ }^{3}$ for the management of osteoid osteomas. This technique offers good long-term pain control with low disease recurrence. ${ }^{4}$

\section{References}

1. Upadhyay $A R$, Desai NC, et al. Role of percutaneous computed tomography-guided radiofrequency ablation in treatment of osteoid osteoma. South Asian J Cancer 2017;6(4):139-140. DOI: 10.4103/sajc. sajc_58_17.

2. Garge S, Keshava SN, et al. Radiofrequency Ablation of Osteoid Osteoma in Common and Technically Challenging Locations in Pediatric Population. Indian J Med Paediatr Oncol 2017;38(3):302-305.

3. Yang J, Li W, et al. Radiofrequency ablation of osteoid osteoma in the atlas: a case report. Interv Neuroradiol 2018 Feb;24(1):88-92.

4. Kulkarni S, Nitin S, et al. CT-guided radiofrequency ablation in osteoid osteoma: result from a tertiary cancer centre in India. Indian J Radiol Imaging 2017;27(3):318-323. 